\title{
TYPICAL INTERSECTIONS OF CONTINUOUS FUNCTIONS WITH MONOTONE FUNCTIONS
}

\author{
M. HEJNÝ
}

(Communicated by Andrew M. Bruckner)

\begin{abstract}
For each parameter $\Phi$ a typical continuous function intersects every monotone function in a $(\Phi)$-uniformly symmetrically porous set.
\end{abstract}

\section{INTRODUCTION AND NOTATION}

This paper generalizes the results of Humke and Laczkovich [1]. The notion of "bilaterally strongly $\Phi$-porosity" in [1] is replaced by the stronger one of “( $\Phi)$-uniformly symmetric porosity" (see Definition 2 and Theorem 3 ). The main result of the paper, Theorem 4, is obtained using an adaptation of the Banach-Mazur game (Theorem 6), and there is a different approach than that used in [1].

Definition 1. Let $\Phi:(0,1) \rightarrow(0,1]$ be a continuous function. A set $E \subset \mathbb{R}$ is said to be bilaterally strongly $\Phi$-porous if for every $x \in E$ there are sequences of intervals $I_{n} \subset(x-1 / n, x) \backslash E$ and $J_{n} \subset(x, x+1 / n) \backslash E$ such that

$$
\lim _{x \rightarrow \infty} \frac{\operatorname{dist}\left(x, I_{n}\right)}{\Phi\left(\left|I_{n}\right|\right)}=\lim _{x \rightarrow \infty} \frac{\operatorname{dist}\left(x, J_{n}\right)}{\Phi\left(\left|J_{n}\right|\right)}=0 .
$$

In [1] Humke and Laczkovich proved the following

Theorem 1. Let $\Phi:(0,1] \rightarrow(0,1]$ be a continuous function. Then a typical continuous function intersects every monotone function in a bilaterally strongly $\Phi$-porous set.

Notation 1 . The family of all continuous increasing functions $\Phi$ on $[0,1]$ for which $\Phi(0)=0$ will be denoted by $G$; such functions will be referred to as porosity indices.

Notation 2. Let $\Phi \in G$ and $k \in \mathbb{N}$. By $R(\Phi, k)$ we will denote the set of all $E \subset \mathbb{R}$ for which there are numbers $a_{k}, b_{k}$ such that for all $x \in E$ the following hold:

(i) $0<a_{k}<b_{k}<k^{-1}$,

Received by the editors February 20,1991 and, in revised form, December 4, 1991.

1991 Mathematics Subject Classification. Primary 26A48, 26A99.

Key words and phrases. Typical continuous function, intersection with monotonous functions, porous set, Banach-Mazur game. 
(ii) $\Phi\left(b_{k}-a_{k}\right)>a_{k}$, and

(iii) $\left[x-b_{k}, x-a_{k}\right] \cap E=\left[x+a_{k}, x+b_{k}\right] \cap E=\varnothing$.

Further let us denote $R(\Phi)=\bigcap_{k=1}^{\infty} R(\Phi, k)$.

Definition 2. Let $\Phi \in G$. Those sets $E \in R(\Phi)$ are said to be ( $\Phi)$-uniformly symmetrically porous.

For our purposes Theorem 1 will be slightly reformulated.

Definition 3. Let $\Psi \in G$. We call a set $E \subset \mathbb{R}(\Psi)$-bilaterally strongly porous if for every $x \in E$ there are sequences of intervals $I_{n} \subset(x-1 / n, x) \backslash E$ and $J_{n} \subset(x, x+1 / n) \backslash E$ such that for every $n \in \mathbb{N}$ both $\operatorname{dist}\left(x, I_{n}\right)<\Psi\left(\left|I_{n}\right|\right)$ and $\operatorname{dist}\left(x, J_{n}\right)<\Psi\left(\left|J_{n}\right|\right)$.

We reformulate Theorem 1 as

Theorem 2. Let $\Psi \in G$. Then the typical continuous function intersects every monotone function in a $(\Psi)$-bilaterally strongly porous set.

In fact, for each continuous function $\Phi:(0,1] \rightarrow(0,1]$ there exists $\Psi_{0} \in G$ such that $\Psi_{0}(x) \leq \Phi(X)$ for $x \in(0,1]$. Let us set $\Psi(x)=x \Psi_{0}(x)$. For $\Psi \in G$ we can find (according to Theorem 2) sequences of intervals $\left\{I_{n}\right\}_{n=1}^{\infty}$ and $\left\{J_{n}\right\}_{n=1}^{\infty}$ such that for every $n \in \mathbb{N}, \operatorname{dist}\left(x, I_{n}\right)<\Psi\left(\left|I_{n}\right|\right)$ and $\operatorname{dist}\left(x, J_{n}\right)<$ $\Psi\left(\left|J_{n}\right|\right)$. Then $\operatorname{dist}\left(x, I_{n}\right)<\Psi\left(\left|I_{n}\right|\right)=\left|I_{n}\right| \Psi_{0}\left(\left|I_{n}\right|\right) \leq n^{-1} \Phi\left(\left|I_{n}\right|\right)$. Similarly $n \operatorname{dist}\left(x, J_{n}\right)<\Phi\left(\left|J_{n}\right|\right)$ and, hence, (a).

Therefore Theorem 1 is a consequence of Theorem 2. The fact that Theorem 2 is a consequence of Theorem 1 is evident.

\section{Proof of Theorem 3}

In this section we will show by Theorem 3 that our main result (Theorem 4) is stronger than the result of Humke and Laczkovich (Theorem 2).

Theorem 3. Let $\Phi$ be the identity function on $[0,1]$. Then for every $\Psi \in G$ there is $E \subset[0,1]$ such that $E$ is $(\Psi)$-bilaterally strongly porous and is not $(\Phi)$-uniformly symmetrically porous.

Proof. Let $\Psi \in G$. The subsequence $\left\{p_{k_{n}}\right\}_{n=1}^{\infty}$ of the sequence $\left\{p_{k}\right\}_{k=1}^{\infty}=$ $\left\{\left(\frac{2}{3}\right)^{k+1}\right\}_{k=1}^{\infty}$ will be defined by induction; set $k_{1}=1$ and suppose $k_{1}, k_{2}, \ldots, k_{n}$ are given. Since $\Psi \in G$, there exists $k_{n+1} \in \mathbb{N}$ such that $\Psi\left(p_{k_{n}}-p_{k_{n+1}}\right)>p_{k_{n+1}}$.

Now denote

$$
\begin{aligned}
E= & \left\{0.5+p_{m}: k_{2 n-1} \leq m \leq k_{2 n}, \quad n \in \mathbb{N}\right\} \cup\{0.5\} \\
& \cup\left\{0.5-p_{m}: k_{2 n} \leq m \leq k_{2 n+1}, n \in \mathbb{N}\right\} .
\end{aligned}
$$

Obviously there is only one point, namely, 0.5 , for which it is necessary to verify ( $\Psi)$-bilaterally strongly porosity. Setting

$$
I_{n}=\left(0.5-p_{k_{2 n-1}}, 0.5-p_{k_{2 n}}\right)
$$

and

$$
J_{n}=\left(0.5+p_{k_{2 n+1}}, 0.5+p_{k_{2 n}}\right),
$$

it is easy to see that the set $E$ is $(\Psi)$-bilaterally strongly porous. Further for each $a \in(0,0.2)$ either $[0.5-2 a, 0.5-a] \cap E \neq \varnothing$ or $[0.5+a, 0.5+2 a] \cap E \neq \varnothing$. Thus the set $E$ is not $(\Phi)$-uniformly symmetrically porous. Q.E.D. 
In Theorem 3 the set $E$ could have been chosen as

$$
\begin{aligned}
E= & \left\{0.25-p_{m}: k_{2 n-1} \leq m \leq k_{2 n}, n \in \mathbb{N}\right\} \\
& \cup\left\{0.25+p_{m}: k_{2 n-1} \leq m \leq k_{2 n}, n \in \mathbb{N}\right\} \\
& \cup\left\{0.75-p_{m}: k_{2 n} \leq m \leq k_{2 n+1}, n \in \mathbb{N}\right\} \\
& \cup\left\{0.75+p_{m}: k_{2 n} \leq m \leq k_{2 n+1}, n \in \mathbb{N}\right\} \\
& \cup\{0.25,0.75\} .
\end{aligned}
$$

Now for each $x \in E$ and each $k \in \mathbb{N}$ there are numbers $a_{k}, b_{k}$ such that (i), (ii), and (iii) of Notation 1 are fulfilled, but the uniformity is not preserved, i.e., there are no sequences $\left\{a_{k}\right\}_{k=1}^{\infty},\left\{b_{k}\right\}_{k=1}^{\infty}$ such that for each $x \in E$ (in our case we put $x=0.25$ or $x=0.75$ ) (i), (ii), and (iii) hold.

The main theorem of this paper reads as follows.

Theorem 4. Let $\Phi \in G$. Then the typical continuous function intersects each monotone function in a $(\Phi)$-uniformly symmetrically porous set.

It is clear that Theorem 2 is a consequence of Theorem 4, but by Theorem 3 we showed that Theorem 2 is not a consequence of Theorem 4 .

\section{Proof of Theorem 2}

Notation 3. Let $f$ be a real function, $g \in C[0,1], x \in \mathbb{R}, \varepsilon>0$. We denote

$$
\begin{gathered}
U(x, \varepsilon)=\{y \in \mathbb{R}:|x-y|<\varepsilon\}, \\
U(g, \varepsilon)=\{\varphi \in C[0,1]:|\varphi(x)-g(x)|<\varepsilon \text { for } x \in[0,1]\}, \\
M_{f, \varepsilon}=\left\{(x, y) \in \mathbb{R}^{2}:|f(x)-y|<\varepsilon\right\},
\end{gathered}
$$

and

$$
\operatorname{gr} f=\left\{(x, f(x)) \in \mathbb{R}^{2}: x \in[0,1]\right\} .
$$

For a set $M \subset \mathbb{R}^{2}$ we denote $P(M)=\{x \in \mathbb{R}$ : there exists $y \in \mathbb{R}$ such that $(x, y) \in M\}$.

We need two lemmas. The first of these is easy to see and is not proved.

Lemma 1. Let $\delta>0, \alpha>0$. Let $r$ be a continuous piecewise linear function on interval $I$, for which $r_{+}^{\prime}(x)<-\alpha$, for all $x \in$ int $I$. Let $f$ be a nondecreasing function. Then there exists an interval of length $2 \delta / \alpha$ which contains the set $P\left(M_{r, \delta} \cap \operatorname{gr} f\right)$.

Lemma 2. Let $U \subset C[0,1]$ be open and nonempty. Then there exists $n \in$ $\mathbb{N}$ such that for an arbitrary $\gamma>0$ there is a function $s \in C[0,1]$ and a number $\delta>0$ such that for each nondecreasing function $f$ there are intervals $J_{1}, J_{2}, \ldots, J_{n}$ for which

(i) $\left|J_{i}\right|<\gamma$ for $i=1,2, \ldots, n$,

(ii) $U(s, \delta) \subset U$, and

(iiii) $P\left(M_{s, \delta} \cap \operatorname{gr} f\right) \subset \bigcup_{i=1}^{n} J_{i}$.

Proof. Obviously there exists a continuous and piecewise linear function $h \in$ $C[0,1]$ and a number $\varepsilon>0$ such that $U(h, \varepsilon) \subset U$. Denote

$$
t=\max \left\{\left|h_{+}^{\prime}(x)\right|: x \in(0,1)\right\},
$$


choose $n_{0} \in \mathbb{N}$ such that

$$
n_{0}>\frac{t+1}{\varepsilon},
$$

and denote $n=2 n_{0}$. Further let $\gamma>0, b=\min \{1 / n, \gamma / 2\}$, and $I=$ $\left[b, 1 / n_{0}\right]$. Let $u_{0}$ be the continuous and piecewise linear function defined as follows:

(a) $u_{0}$ is periodic with the period $n_{0}^{-1}$;

(b) $u_{0}(0)=-\frac{\varepsilon}{2}, u_{0}(b)=\frac{\varepsilon}{2}, u_{0}\left(n_{0}^{-1}\right)=-\frac{\varepsilon}{2}$; and

(c) $u_{0}$ is linear on $[0, b]$ and on $I$.

Let us denote $u(x)=u_{0}(x)_{\mid[0,1]}$, and let $\delta>0$ be such that

$$
2 \delta<\min \{\gamma, \varepsilon\} \text {. }
$$

Suppose $f$ is nondecreasing. Denote $s=h+u$ and $J_{i+1}=\left[i n_{0}^{-1}, i n_{0}^{-1}+b\right]$ for $i=0,1, \ldots, n_{0}-1$. The upcoming construction of intervals $J_{n_{0}+1}, J_{n_{0}+1}, \ldots$, $J_{n}$ will use Lemma 1.

From (2) and the definition of the function $u(x)$ it follows that $u^{\prime}(x)=$ $-\varepsilon /\left(n_{0}^{-1}-b\right)<-\varepsilon n_{0}<-t-1$ for $x \in$ int $I$. Thus by (1)

$$
s_{+}^{\prime}(x)=u_{+}^{\prime}(x)+h_{+}^{\prime}(x)<-t-1+t=-1
$$

for $x \in \operatorname{int} I$. Denote $s_{1}=s_{\mid I}$. From (3) and the fact that $s_{+}^{\prime}(x)<-1$ for $x \in$ Int $I$ it follows from Lemma 1 that there is an interval $J_{n_{0}+1}$ of the length less than $\gamma$ for which $P\left(M_{s_{1}, \delta} \cap \operatorname{gr} f\right) \subset J_{n_{0}+1}$. Similarly we define the intervals $J_{n_{0}+i}$ for $i=2,3, \ldots, n_{0}$. Thus for $s_{i}=s_{\mid\left[(i-1) / n_{0}+b, 1 / n_{0}\right]}$ it follows that $P\left(M_{s_{i}}, \delta \cap \operatorname{gr} f\right) \subset J_{n_{0}+i}$. Conclusions (i) and (iii) follow directly from the definition of the intervals $J_{1}, \ldots, J_{n}$. From (3) and the inequality $|u(x)| \leq \varepsilon / 2$ it follows that $U(s, \delta) \subset U(h, \varepsilon)$; but then $U(h, \varepsilon) \subset U$ and conclusion (ii) follows. Q.E.D.

To prove Theorem 4 we introduce the Banach-Mazur game: Assume that $X$ is a complete metric space and $B \subset X$. The Banach-Mazur game is played by two players, (A) and (B). In the first step, (A) chooses an open and nonempty set $U_{1} \subset X$, and (B) chooses an open and nonempty set $V_{1} \subset U_{1}$. In the $n$th step (A) chooses an open and nonempty set $U_{n} \subset V_{n-1}$ and (B) chooses an open and nonempty set $V_{n} \subset U_{n}$. This defines a nonincreasing sequence of open sets. If $\bigcap_{i=1}^{\infty} V \subset B$ then (B) wins. In the opposite case (A) wins.

Theorem 5 (see [1]). In the Banach-Mazur game there is a winning strategy for the player (B) if and only if the set $B$ is residual in $X$.

Further the Banach-Mazur game will be looked at with respect to the space $X=C[0,1]$.

Definition 4. A nonempty family $P$ of subsets of $\mathbb{R}$ is called a family of small sets if the relation $A \in P$ and $B \subset A$ yields $B \in P$.

Notation 4. Let $P$ be a family of small sets. The Banach-Mazur game for which $B=\{\varphi \in C[0,1]: P(\operatorname{gr} f \cap \operatorname{gr} \varphi) \in P$ for every nondecreasing function $f\}$ will be denoted by $\mathrm{BM}(P)$.

Further we will use the $\mathrm{F}(P)$ game, which is described as follows: The $\mathrm{F}(P)$ game is played by two players, (A) and (B). In the first step, (A) chooses a 
number $n_{1} \in \mathbb{N}$ and (B) chooses a real positive number $\gamma_{1}>0$. In the $k$ th step (A) chooses a number $n_{k} \in \mathbb{N}$ and (B) chooses a real positive number $\gamma_{k}>0$. This defines a sequence $n_{1}, \gamma_{1}, n_{2}, \gamma_{2}, \ldots$ If for every sequence $\left\{T_{k}\right\}_{k=1}^{\infty}$ of sets $T_{k}=\bigcup_{i=1}^{n_{k}} I_{i}^{k}$, where $I_{i}^{k}\left(i=1,2, \ldots, n_{k}\right)$ are intervals shorter than $\gamma_{k}$, $\bigcap_{k=1}^{\infty} T_{k} \in P$ holds, then (B) wins. In the opposite case (A) wins.

Lemma 3. Let $P$ be a family of small sets. If there is a winning strategy for the player $(\mathrm{B})$ in the $\mathrm{F}(P)$ game then there is in the $\mathrm{BM}(P)$ game as well.

Proof. Suppose that the $\operatorname{BM}(P)$ game up to the $k$ th step of $(\mathrm{A})$ is given by the sequence $U_{1} \supset V_{1} \supset \cdots \supset U_{k}$, and suppose that the $\mathrm{F}(P)$ game up to the $(k-1)$ th step of $(\mathrm{B})$ is given by the sequence $n_{1}, \gamma_{1}, \ldots, n_{k-1}, \gamma_{k-1}$ and that (B) has used a winning strategy. Now for $U_{k} \subset C[0,1]$ by Lemma 2 we find $n_{k} \in \mathbb{N}$. Then with respect to the winning strategy of $(\mathrm{B})$ in the $\mathrm{F}(P)$ game for $n_{k} \in \mathbb{N}$ we obtain a number $\gamma_{k}>0$. For $U_{k}, n_{k}$, and $\gamma_{k}$ by Lemma 2 we find a function $s \in C[0,1]$ and a number $\delta_{k}>0$. Now put $V_{k}=U\left(s_{k}, \delta_{k}\right)$ as the $k$ th step of (B). From Lemma 2 we have $V_{k} \subset U_{k}$.

We are going to show that $(\mathrm{B})$ wins in this $\mathrm{BM}(P)$ game. If $\bigcap_{i=1}^{\infty} V_{i}=\varnothing$ there is nothing to be proved. Let $s \in \bigcap_{i=1}^{\infty} V_{i}$ and let $f$ be a nondecreasing function. Denote $E=P(\operatorname{gr} f \cap \operatorname{gr} s)$. According to Lemma 2 for each $k \in \mathbb{N}$ there are intervals $I_{1}^{k}, I_{2}^{k}, \ldots, I_{n_{k}}^{k}$ such that $\left|I_{1}^{k}\right|<\gamma_{k}, i=1,2, \ldots, n_{k}$, and

$$
P\left(\operatorname{gr} f \cap M_{s_{k}, \delta_{k}}\right) \subset T_{k} \quad \text { where } T_{k}=\bigcup_{i=1}^{n_{k}} I_{i}^{k} .
$$

Obviously $E \subset P\left(\operatorname{gr} f \cap M_{s_{k}}, \delta_{k}\right)$, and regarding (4) we have

$$
E \subset \bigcap_{k=1}^{\infty} T_{k}
$$

Since (B) used the winning strategy in the $\mathrm{F}(P)$ game, it follows that $\bigcap_{i=1}^{\infty} T_{k}$ $\in P$. From (5) and the fact that $P$ is a family of small sets we get $E \in P$. Hence $(B)$ wins in the $\operatorname{BM}(P)$ game as well. Q.E.D.

Notation 5. Let $P$ be a family of small sets. Then we denote

$$
\begin{aligned}
& W_{P}=\{\varphi \in C[0,1]: P(\operatorname{gr} f \cap \operatorname{gr} \varphi) \in P \text { for each } f \text { monotone }\}, \\
& W_{P}^{-}=\{\varphi \in C[0,1]: P(\operatorname{gr} f \cap \operatorname{gr} \varphi) \in P \text { for each } f \text { nonincreasing }\}, \\
& W_{P}^{+}=\{\varphi \in C[0,1]: P(\operatorname{gr} f \cap \operatorname{gr} \varphi) \in P \text { for each } f \text { nondecreasing }\} .
\end{aligned}
$$

Lemma 4. Let $P$ be a family of small sets. The set $W_{P}^{+}$is residual if and only if the set $W_{P}^{-}$is residual.

Proof. Obviously it is enough to find a homeomorphism $H$ from $C[0,1]$ on to $C[0,1]$ such that $H\left(W_{P}^{+}\right)=W_{P}^{-}$. Define $H(\varphi)=-\varphi$ for $\varphi \in C[0,1]$. Let $\varphi \in W_{P}^{+}$, and let $f$ be nonincreasing. Then $P(\operatorname{gr} f \cap \mathrm{gr}-\varphi)=P(\mathrm{gr}-f \cap \operatorname{gr} \varphi) \in$ $P$. Thus $-\varphi \in W_{P}^{-}$. Similarly $\varphi \in W_{P}^{-}$yields $-\varphi \in W_{P}^{+}$. Q.E.D.

Lemma 5. Let $P$ be a family of small sets such that the set $W_{P}^{+}$is residual. Then $W_{P}$ is also residual in $C[0,1]$.

Proof. According to Lemma 4 the set $W_{P}=W_{P}^{+} \cap W_{P}^{-}$is residual. Because $W_{P}=W_{P}^{+} \cap W_{P}^{-}, W_{P}$ is thus residual also. Q.E.D. 
Theorem 6. Let $P$ be a family of small sets. If there is a winning strategy for the player $(\mathrm{B})$ in the $\mathrm{F}(P)$ game then the set $W_{P}$ is residual in $C[0,1]$.

Proof. According to Lemma 3 there is a winning strategy for (B) also in the $\operatorname{BM}(P)$ game, and with respect to Theorem 5 the set $W_{P}^{+}$is residual. Theorem 6 now follows from Lemma 5. Q.E.D.

Proof of Theorem 4. Let $\Phi \in G$. We need to show that $W_{R(\Phi)}$ is residual. To do that it is enough to verify the assumptions of Theorem 5. Obviously it is enough to find the winning strategy for player $(\mathrm{B})$ in the $\mathrm{F}(R(\Phi))$ game. Let the $k$ th step of player (A) be given by the number $n_{k} \in \mathbb{N}$. add We are going to find a number $\gamma_{k}>0$ as a $k$ th step of player (B). The set $A=$ $\left\{\left(x_{1}, x_{2}, \ldots, x_{n_{k}}\right) \in \mathbb{R}^{n_{k}}: 0 \leq x_{1} \leq x_{2} \leq \cdots \leq x_{n_{k} \leq 1}\right\}$, as a subset of the metric space $\left(\mathbb{R}^{n_{k}}, \rho\right)$ with maxim metric $\rho$ (i.e., $\rho(x, y)=\max \left\{\left|x_{i}-y_{i}\right|: i=\right.$ $\left.1,2, \ldots, n_{k}\right\}$ where $x=\left(x_{1}, x_{2}, \ldots, x_{n_{k}}\right)$ and $\left.y=\left(y_{1}, y_{2}, \ldots, y_{n_{k}}\right)\right)$, is compact. For $x=\left(x_{1}, x_{2}, \ldots, x_{n_{k}}\right) \in A$ we define function $F(x)=\sup \{\varepsilon>$ $\left.0: \bigcup_{i=1}^{n_{k}} U\left(x_{1}, \varepsilon\right) \in R(\Phi, k)\right\}$. Further we will prove that there is a number $\gamma_{k}>0$ such that

$$
F(x)>\gamma_{k} \quad \text { for all } x \in A \text {. }
$$

Because $A$ is compact, in order to prove (6) it is sufficient to prove the following two assertions:

$$
F(x)>0 \text { for all } x \in A,
$$

and

$$
F \text { is continuous on } A \text {. }
$$

The proof of (7) will be divided into two cases.

I. If $x_{1}=x_{n_{k}}$, denote $b_{k}=1 /(k+1)$. Because $\Phi \in G$, there exists $a_{k}>0$ such that $\Phi\left(b_{k}-a_{k}\right)>a_{k}$. For $\varepsilon=2^{-1} a_{k}$ obviously $\bigcup_{i=1}^{n_{k}} U\left(x_{1}, \varepsilon\right) \in R(\Phi, k)$.

II. If $x_{1} \neq x_{n_{k}}$, denote $d=\min \left\{\left|x_{i}-x_{j}\right|>0: i, j=1,2, \ldots, n_{k}\right\}$ and $b_{k}=\min \{d / 2,1 /(k+1)\}$. It is clear that there is a number $a_{k}>0$ such that $\Phi\left(b_{k}-a_{k}\right)>a_{k}$. For $\varepsilon=2^{-1} a_{k}$ it is $\bigcup_{i=1}^{n_{k}} U\left(x_{i}, \varepsilon\right) \in R(\Phi, k)$.

To prove $(8)$ it is sufficient to show

Lemma 6. Let $x=\left(x_{1}, x_{2}, \ldots, x_{n_{k}}\right) \in A, y=\left(y_{1}, y_{2}, \ldots, y_{n_{k}}\right) \in A$, and $\rho(x, y)<\delta$. Then $\mathrm{F}(x)+\delta \geq \mathrm{F}(y)$.

Proof. If $\mathrm{F}(y) \leq \delta$ then clearly Lemma 6 holds. If $\mathrm{F}(y)>\delta$ then there exists $\varepsilon>\delta$ such that $\bigcup_{i=1}^{n_{k}} U\left(y_{i}, \varepsilon\right) \in R(\Phi, k)$. From $\rho(x, y)<\delta$ we have $\bigcup_{i=1}^{n_{k}} U\left(x_{i}, \varepsilon-\delta\right) \subset \bigcup_{i=1}^{n_{k}} U\left(y_{i}, \varepsilon\right)$, and since $R(\Phi, k)$ is a family of small sets, we obtain $\bigcup_{i=1}^{n_{k}} U\left(x_{i}, \varepsilon-\delta\right) \in R(\Phi, k)$. Q.E.D.

To finish the proof of Theorem 4 we will show that in the described game given by $n_{1}, \gamma_{1}, n_{2}, \gamma_{2}, \ldots$ (B) wins.

Let there be a sequence $\left\{T_{k}\right\}_{k=1}^{\infty}, T_{k}=\bigcup_{i=1}^{n_{k}} I_{i}^{k}$, where intervals $I_{i}^{k} \quad(i=$ $\left.1,2, \ldots, n_{k}\right)$ satisfy $\left|I_{i}^{k}\right|<\delta_{k}$. Then there exists $x=\left(x_{1}, x_{2}, \ldots, x_{n_{k}}\right) \in A$ such that $T_{k} \subset \bigcup_{i=1}^{n_{k}} U\left(x_{i}, \gamma_{k}\right)$. With respect to (6) and to the fact that $R(\Phi, k)$ is a family of small sets we obtain $T_{k} \in R(\Phi, k)$. Hence $\bigcap_{i=1}^{\infty} T_{i} \in R(\Phi, k)$, and also $\bigcap_{i=1}^{\infty} T_{i} \in \bigcap_{i=1}^{\infty} R(\Phi, i)=R(\Phi)$. Q.E.D.

It is not very difficult to show that Theorem 4 yields the following assertion. 
Let $\Phi \in G$. Then a typical continuous function intersects every Lipschitz function in $(\Phi)$-uniformly symmetrically porous set.

\section{ACKNOWLEDGMENT}

The results in this paper are part of my diploma thesis (Charles University of Prague, 1989) written under the direction of Professor L. Zajiček. I thank him for his help and encouragement.

\section{REFERENCES}

1. P. D. Humke and M. Laczkovich, Typical continuous functions are virtually nonmonotone, Proc. Amer. Math. Soc. 94 (1985), 244-248.

2. __ Contributions to the theory of games, vol. 3, Ann. Math. Stud., no. 39, Princeton Univ. Press, Princeton, NJ, 1957, pp. 159-163.

Department of Applied Mathematics, Faculty of Matematics and Physics, Comenius University, Mlynská Dolina, 842 15 Bratislava, Czechoslovakia

E-mail address: hejny@mff.uniba.cs 